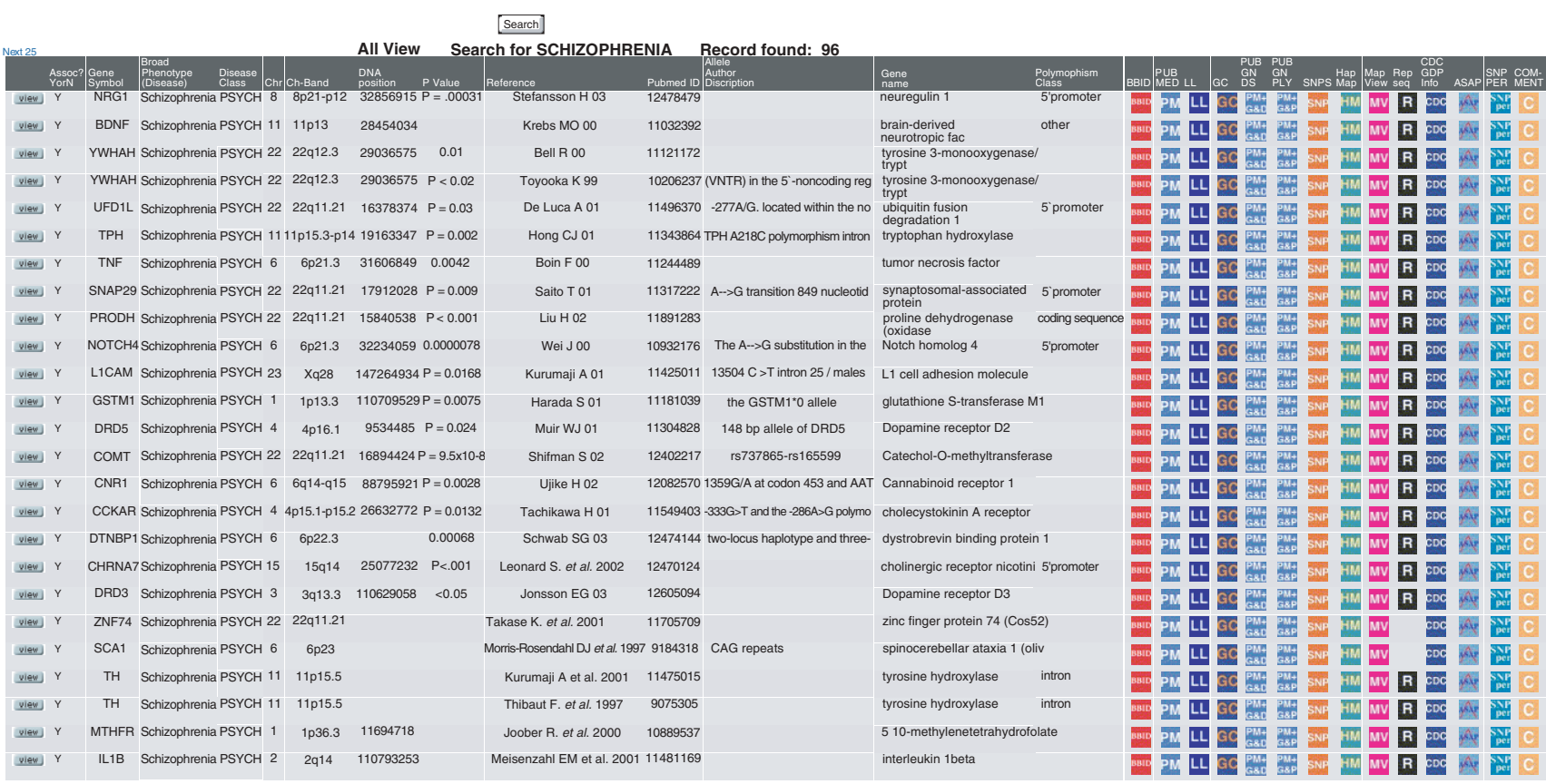

Figure 1 A simple search of positive associations for the disease schizophrenia. Fields in this view include Official Gene Symbol, Disease Phenotype, Disease Class, Chromosome, Chromosome Band, Genomic DNA Position, P Value, Reference, PubMed ID and Allele.

overt similarities at a clinical level, the genetic factors that contribute to those diseases may be shared at a molecular level ${ }^{8,9}$. The development of a hierarchy of phenotypes, from broad to specific, may allow classification of diseases, subphenotypes and molecular parameters of disease and their relationship to complex traits.

GAD is an archive of published genetic association studies that provides a comprehensive, public, web-based repository of molecular, clinical and study parameters for $>5,000$ human genetic association studies at this time. This approach will allow the systematic analysis of complex common human genetic disease in the context of modern high-throughput assay systems and current annotated molecular nomenclature.

Kevin G Becker ${ }^{1}$, Kathleen C Barnes ${ }^{2}$, Tiffani J Bright ${ }^{1}$ \& S Alex Wang ${ }^{3}$

${ }^{1}$ Gene Expression and Genomics Unit, 333 Cassell Drive, National Institute on Aging, National Institutes of Health, Baltimore, Maryland 21224, USA. ${ }^{2}$ Johns Hopkins Asthma and Allergy Center, Johns Hopkins University, 5501 Hopkins Bayview Circle, Baltimore, Maryland 21224, USA.

${ }^{3}$ Division of Computational Bioscience, Center for Information Technology, National Institutes of
Health, Bethesda, Maryland 20892, USA. Correspondence should be addressed to K.G.B. (beckerk@grc.nia.nih.gov).

1. Thorisson, G.A. \& Stein, L.D. Nucleic Acids Res. 31, 124-127 (2003).

2. Sherry, S.T. et al. Nucleic Acids Res. 29, 308-311 (2001).

3. Hamosh, A. et al. Nucleic Acids Res. 30, 52-55 (2002).

4. Coope, D.N., Nussbaum, R.L. \& Krawczak, M. Hum Genet. 110, 207-208 (2002).

5. Anonymous. Nat. Genet. 22, 1-2 (1999)

6. Dahlman, I. et al. Nat. Genet. 30, 149-150 (2002).

7. Funalot, B., Varenne, O. \& Mas, J.L. Nat. Genet. 36, 3 (2004).

8. Mira, M.T. et al. Nature 427, 636-640 (2004).

9. Becker, K.G. Med. Hypotheses 62, 309-317 (2004)

\title{
Is mismatch repair really required for ionizing radiation-induced DNA damage signaling?
}

\section{To the editor:}

The MMR system has evolved to increase the fidelity of DNA replication and homologous recombination ${ }^{1}$. MMR is also implicated in the processing of other types of DNA damage, as mammalian cells with defective MMR are tolerant to $\mathrm{S}_{\mathrm{N}} 1$ type methylating agents such as $\mathrm{N}$-methyl- $\mathrm{N}^{\prime}$ nitro- $\mathrm{N}$-nitrosoguanidine and to 6thioguanine and cisplatin ${ }^{2}$.
Reports describing the differential sensitivity of MMR-proficient and -deficient cells to ionizing radiation raised some controversy, as MMR-deficient cells were found to be slightly more resistant to ionizing radiation in some laboratories ${ }^{3}$ but either equally ${ }^{4}$ or less resistant ${ }^{5}$ in others. The survival differences were also questioned, because MMR status was reported to affect the length of the G2-M checkpoint rather than cell viability ${ }^{6}$. A report by Brown et al. ${ }^{7}$ has reopened this discussion by describing the requirement of a functional MMR system for activating the S-phase checkpoint and signaling of ionizing radiation-induced damage.

The aforementioned studies used matched MMR-proficient and -deficient mouse or human cell lines. Given that the establishment of these lines involved long periods of growth in cell culture, and that the MMR-deficient 
a

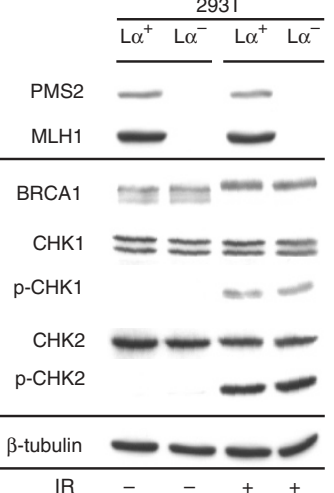

b

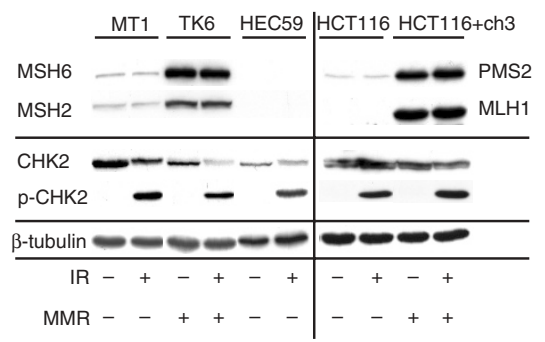

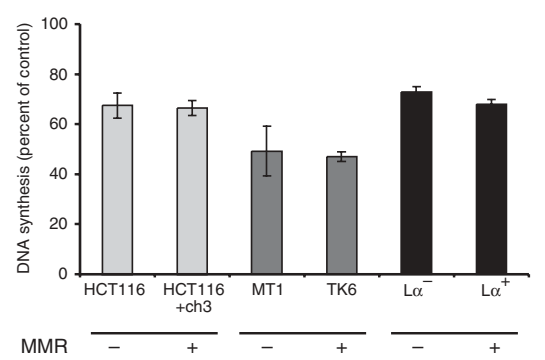

Figure 1 The response of human cells to ionizing radiation is independent of their MMR status. (a) MMR-proficient 293T L $\alpha^{+}$and MMR-deficient 293T L $\alpha^{-}$cells were treated with ionizing radiation (IR; 4 Gy) and the extracts were prepared $1 \mathrm{~h}$ later. They were immunoblotted with antibodies against MLH1 and PMS2 (upper panel) to confirm the MMR status of the cells and with antibodies against BRCA1, CHK1, phosphorylated CHK1 (p-CHK1), CHK2 and phosphorylated CHK2 (p-CHK2; lower panel) to examine the extent of ionizing radiation--induced DNA damage signaling. No notable differences in post-translational modification of the latter polypeptides were observed. $\beta$-tubulin was used as a loading control. (b) Response of the lymphoblastoid lines TK6 (MMR-proficient) and MT1 (MMR-deficient; derived from TK6, carries mutations in both alleles of MSH6), the colon cancer lines HCT116 (MMR-deficient; carries mutations in both alleles of MLH1) and HCT116+ch3 (MMR-proficicent; derived from HCT116 by transfer of chromosome 3, which carries the wild-type MLH1) and HEC59 cells (MMR-deficient; derived from a human endometrial tumor; carry mutations in both alleles of MSH2) to ionizing radiation (IR; 5 Gy). The MMR proteins MSH2 and MSH6, immunoblotted with MLH1 and PMS2, confirmed the MMR status of these lines (top). The phosphorylation status of the checkpoint kinase CHK2 (p-CHK2) is shown (bottom). Preparation of cell extracts and immunoblotting procedures were described earlier ${ }^{8}$. (c) Effect of ionizing radiation on DNA synthesis. Cells were prelabeled with ${ }^{14} \mathrm{C}$-thymidine for $24 \mathrm{~h}$, incubated in isotope-free medium for an additional $24 \mathrm{~h}$ and exposed to ionizing radiation (4 Gy). They were pulse-treated $45 \mathrm{~min}$ later with ${ }^{3} \mathrm{H}$-thymidine for $15 \mathrm{~min}$ and collected. DNA synthesis was estimated as a ratio of ${ }^{3} \mathrm{H} /{ }^{14} \mathrm{C}$ counts and expressed as a percentage of control values. The cells were not contact-inhibited during the course of the experiment. Results shown are the mean \pm s.e. of four independent experiments. Similar results were obtained when the MT1-TK6 cell pair was pulse-labeled after 30 or 60 min. Although no MMR-dependent differences were observed, there were substantial differences between cells of different origin. Whereas the lymphoblastoid MT1-TK6 cells showed the strongest inhibition of DNA synthesis, the S-phase checkpoint was activated only weakly in the epithelial 293T L $\alpha^{+}$and 293T L $\alpha^{-}$cells. This is probably linked with expression of the SV40 large T antigen in the latter cells, which is known to interfere with S-phase checkpoint effectors ${ }^{10}$.

cells have a mutator phenotype, we considered the possibility that the differential responses of these cells to DNA-damaging agents could be linked to phenotypic traits other than MMR. We therefore examined the response to ionizing radiation of the strictly isogenic 293T $\mathrm{La}^{+}$and 293T $\mathrm{L}^{-}$cell pair, in which the MMR-proficient 293T L $\alpha^{+}$cells differ from the MMR-deficient $293 \mathrm{~T}$ L $\alpha^{-}$cells solely by expression of the MMR protein MLH1 (ref. 8) and in which switching the MMR status does not involve clonal selection.

We exposed the 293T $\mathrm{L}^{+}$and 293T $\mathrm{L} \alpha^{-}$ cells to ionizing radiation and monitored their viability and progress through the cell cycle for $72 \mathrm{~h}$. Ionizing radiation arrested both cell types in G2-M after $\sim 20 \mathrm{~h}$, and we observed no differences in clonogenic survival (data not shown). We also observed no MMR-dependent differences in phosphorylation of the checkpoint kinases CHK1 and CHK2 (activation of which is required for triggering the arrest; Fig. 1a), of NBS1 (data not shown) or of BRCA1 (implicated in the processing of ionizing radiation-induced strand breaks; Fig. 1a). Thus, the MMR status per se did not affect DNA damage signaling in these cells.

We observed no MMR-dependent differences in early post-translational modification of CHK2 in other matched MMR-proficient and -deficient cell line pairs (Fig. 1b), some of which were also used by Brown and colleagues ${ }^{7}$. When we measured radiation-resistant DNA synthesis in some of these cell lines, we also observed no MMRdependent differences (Fig. 1c). Although the clones used in our laboratory may not be identical to those examined by Brown et al. ${ }^{7}$, analysis of extracts of our cell lines showed that MMR protein levels (Fig. 1a,b) and MMR capacity measured by an in vitro MMR assay $^{8}$ (data not shown) correlated with their MMR status.

Ionizing radiation induces different types of damage in DNA. The most common by far is oxidation and fragmentation of DNA bases, and the MMR system is involved in

processing 8-oxoguanosines (GOs) incorporated into DNA during replication ${ }^{9}$. This type of damage could only signal during the S phase, however, which is inconsistent with the experimental findings, given that the cells analyzed by Brown et al. ${ }^{7}$ were confluent. It seems more likely that any differences in ionizing radiation-induced DNA damage signaling should be linked with the processing of double-strand breaks, the most deleterious kinds of ionizing radiation-induced DNA damage, as they are processed by recombination, a process in which MMR is involved $^{1}$. Because we observed no differences in our strictly isogenic cell system, however, MMR does not seem to be required to repair ionizing radiation-induced cytotoxic doublestrand breaks. This implies that the small differences in response to ionizing radiation described by others were linked either with small variations in experimental procedures or with phenotypic traits of MMR-deficient cells other than MMR.

\section{Petr Cejka, Lovorka Stojic, Giancarlo Marra \&} Josef Jiricny

Institute of Molecular Cancer Research, University of Zurich, August Forel-Strasse 7, CH-8008 Zurich, Switzerland. Correspondence should be addressed to J.J. (jiricny@imr.unizh.ch).

1. Harfe, B.D. \& Jinks-Robertson, S. Annu. Rev. Genet. 34, 359-399 (2000).

2. Karran, P. Carcinogenesis 22, 1931-1937 (2001).

3. Zeng, M. et al. Cancer Res. 60, 4889-4893 (2000).

4. Aquilina, G., Crescenzi, M. \& Bignami, M. Carcinogenesis 20, 2317-2326 (1999).

5. Franchitto, A. et al. Oncogene 22, 2110-2120 (2003).

6. Yan, T. et al. Cancer Res. 61, 8290-8297 (2001).

7. Brown, K.D. et al. Nat. Genet. 33, 80-84 (2003).

8. Cejka, P. et al. EMBO J. 22, 2245-2254 (2003).

9. Colussi, C. et al. Curr. Biol. 12, 912-918 (2002).

10. Lavia, P., Mileo, A.M., Giordano, A. \& Paggi, M.G. Oncogene 22, 6508-6516 (2003). 


\section{-In reply}

Work from our laboratories showed that MMR-deficient cells had defective activation of the S-phase checkpoint and that this correlated with diminished activation of Chk2 in response to ionizing radiation ${ }^{1}$. Cejka et al. now report that MMR deficiency does not result in these defects. MMR deficiency results in a mutator phenotype; hence, it is conceivable that secondary mutations, which either positively or negatively effect cellular response to genotoxic events, could be responsible for these conflicting results. Differences in cell strains, culture or treatment conditions could also contribute to these contrasting findings. In agreement with our findings, however, Franchitto et al. ${ }^{2}$ also observed defective ionizing radiation-induced phosphorylation of Chk2 in Msh2-deficient mouse embryonic fibroblasts (MEFs).

Although the engineered MMR-inducible cell line used by Cejka et al. offers a technical improvement over the MMR-deficient tumor lines complemented by stable introduction of large fragments of human chromosomes used in many studies, including ours, the isogenic 293T $\mathrm{L}^{+}$and 293 $\mathrm{T} \alpha^{-}$lines are derivatives of the transformed human embryonic kidney line 293T, which is MMR-deficient due to epigenetic silencing of the MLH1 promoter ${ }^{3}$. Thus, it cannot be strictly ruled out that these isogenic lines, like other commonly used MMR-deficient tumor lines, contain other deleterious mutations.

Defects in cell cycle arrest and apoptosis linked to MMR deficiency are most prominently seen in response to $S_{N} 1$ alkylators such as $\mathrm{N}$-methyl- $\mathrm{N}^{\prime}$-nitro-Nnitrosoguanidine and methyl-nitrosourea, the methylating compound temozolomide, the base analog 6-thioguanine and cisplatin. But it may be premature to conclude that MMR does not have any role in ionizing radiation-induced DNA damage signaling. For example, Franchitto et al. ${ }^{2}$ reported that Msh2 was required to sustain G2 arrest in response to ionizing radiation, a finding that is in agreement with studies conducted on MMR-deficient human tumor lines ${ }^{4,5}$. Furthermore, Zeng et al. ${ }^{6}$ showed that Pms2-deficient MEFs had significantly less apoptosis after irradiation than wild-type MEFs, and similar results were obtained in a study that examined Msh2-deficient mouse embryonic stem cells ${ }^{7}$.

Ionizing radiation induces a wide spectrum of damage in DNA, including base oxidation and damage to the phosphodiester backbone resulting in single- and double-strand breaks. We stated that we were unsure of the exact nature of the ionizing radiation-induced lesion(s) recognized by MMR but suggested that the presence of the oxidized purine GO could trigger assembly of an MMR complex. This possibility is supported, in part, by the observation that MSH2-MSH6 heterodimers bind to both GO:C and postreplicative GO:A base pairs in vitro ${ }^{8}$. A recent report indicates that oxidation of free nucleotide pools is an important source of $\mathrm{GO}^{9}$ but does not exclude the possibility that oxidation of guanine to GO occurs in situ in DNA.
Moreover, the work of Chen and colleagues indicates that GO is detectable in genomic DNA shortly after irradiation of mitotically synchronized and asynchronous cells ${ }^{10}$. As our S-phase checkpoint assay positively indicated DNA synthesis in irradiated cells (as judged by uptake of radioactively labeled thymidine), we cannot rule out either GO incorporation into the genome as a result of postirradiation replication or oxidation in situ. A more comprehensive understanding of a potential function for MMR in response to ionizing radiation-induced DNA damage and the nature of the lesions that trigger such response will require further investigation.

Kevin D Brown ${ }^{1}$ \& R Baskaran ${ }^{2}$

${ }^{1}$ Department of Biochemistry and Molecular Biology and the Stanley S. Scott Cancer Center, Louisiana State University Health Sciences Center, New Orleans, Louisiana, USA.

${ }^{2}$ Department of Molecular Genetics and Biochemistry, University of Pittsburgh Medical Center, Pittsburgh, Pennsylvania, USA. Correspondence should be addressed to R.B. (bask@mgb.pitt.edu).

1. Brown, K.D. et al. Nat. Genet. 33, 80-84 (2003).

2. Franchitto, A. et al. Oncogene 22, 2110-2120 (2003).

3. Cejka, P. et al. EMBO J. 22, 2245-2254 (2003).

4. Yan, T. et al. Cancer Res. 61, 8290-8297 (2001).

5. Davis, T.W. et al. Cancer Res. 58, 767-778 (1998).

6. Zeng, M. et al. Cancer Res. 60, 4889-4893 (2000).

7. DeWeese, T.L. et al. Proc. Natl. Acad. Sci. USA 95, 11915-11920 (1998)

8. Ni, T.T., Marsischky, G.T. \& Kolodner, R.D. Mol. Cel/4, 439-444 (1999).

9. Colussi, C. et al. Curr. Biol. 12, 912-918 (2002).

10. Chen, S.K., Tsai, M.H., Hwang, J.J., Chang, W.P. \& Chan, W.P. Radiat. Res. 155, 832-836 (2001). 